\title{
La place de la concertation dans la mise en œuvre de projets urbains durables à travers l'exemple de l'agglomération parisienne
}

Territoires, acteurs, représentations

Hélène Sirota-Chelzen

\section{OpenEdition}

Journals

Édition électronique

URL : https://journals.openedition.org/cdg/5267

DOI : $10.4000 /$ cdg. 5267

ISSN : $2107-7266$

Éditeur

UMR 245 - CESSMA

Référence électronique

Hélène Sirota-Chelzen, « La place de la concertation dans la mise en œuvre de projets urbains

durables à travers l'exemple de l'agglomération parisienne », Carnets de géographes [En ligne], 14 |

2020, mis en ligne le 15 décembre 2020, consulté le 20 mai 2021. URL : http://

journals.openedition.org/cdg/5267 ; DOI : https://doi.org/10.4000/cdg.5267

Ce document a été généré automatiquement le 20 mai 2021.

\section{(c)}

La revue Carnets de géographes est mise à disposition selon les termes de la Licence Creative Commons Attribution - Pas d'Utilisation Commerciale - Pas de Modification 4.0 International. 


\title{
La place de la concertation dans la mise en œuvre de projets urbains durables à travers l'exemple de l'agglomération parisienne
}

\author{
Territoires, acteurs, représentations
}

Hélène Sirota-Chelzen

1 Depuis la conférence des Nations-Unies de Rio en 1992 au cours de laquelle ont été affirmés les principes du développement durable, la concertation est devenue une pratique politique et sociale incontournable. En effet, en réunissant une pluralité d'acteurs, les processus participatifs offrent un moment et un lieu où se posent des enjeux de gouvernance, de gestion territoriale et de durabilité urbaine. Les processus participatifs répondent d'une part, à une dynamique bottom-up, c'est-à-dire à une demande des citoyens de participer au débat public, à la prise de décision, à la gestion locale et à la fabrique de la ville, et s'inscrivent d'autre part dans une injonction topdown où un cadre juridique porte les objectifs de durabilité. Les habitants sont de plus en plus sollicités pour s'investir dans des dispositifs participatifs. La thèse cherche à répondre à la question suivante: à quelles conditions, les habitants, à travers les dispositifs participatifs, deviennent-ils des acteurs de leur territoire? Mon hypothèse de recherche est que les dispositifs participatifs transforment les habitants en leur permettant de développer un pouvoir et une capacité d'agir. En développant leur expertise les habitants s'impliquent davantage dans les processus de décision qui concernent leur territoire.

2 Le choix des terrains s'est porté sur la Métropole du Grand Paris et plus particulièrement sur la banlieue parisienne; plusieurs projets durables de la Ville de Paris ayant déjà été étudiés. Sous l'effet de la métropolisation, ces communes vivent actuellement une métamorphose, entre permanence d'une population de l'ex-banlieue rouge et l'arrivée de classes sociales beaucoup plus variées, témoignant d'une gentrification de ces territoires proches du cœur parisien et d'un rejet des classes 
populaires. La métropolisation du territoire se manifeste par une concentration démographique, une concentration des pouvoirs de commandement, tandis que l'application des principes de durabilité urbaine, comme la végétalisation de la ville et l'implication des habitants dans la fabrique de la ville, deviennent obligatoires. Il s'agissait donc d'examiner comment la métropolisation du territoire et la singularité des territoires de l'ex-banlieue rouge affectent les dispositifs participatifs. L'observation a notamment concerné des projets ordinaires: PLU, écoquartier, etc. (figure 1). La méthodologie mise en œuvre s'est appuyée sur l'observation de réunions publiques organisées par les municipalités lors de projets urbains soumis à la concertation. Six territoires, sept projets et trente et un dispositifs participatifs ont été étudiés (figure 1). À l'occasion de ces réunions et des ateliers a été collectée, sur place et sur internet, une documentation concernant le projet et le diagnostic urbain élaborés par les porteurs de projet (responsables politiques et professionnels de l'urbanisme). Les dispositifs participatifs qui ont donné lieu à des réunions publiques ont été enregistrées et les échanges ont été retranscrits. L'observation des réunions a également permis d'étudier la disposition des acteurs dans l'espace, et ce que cette microgéographie donne à voir des rapports de pouvoir et des statuts entre les acteurs.

Figure 1. Le choix des terrains : des communes de l'ex-banlieue rouge parisienne à l'épreuve de projets urbains durables

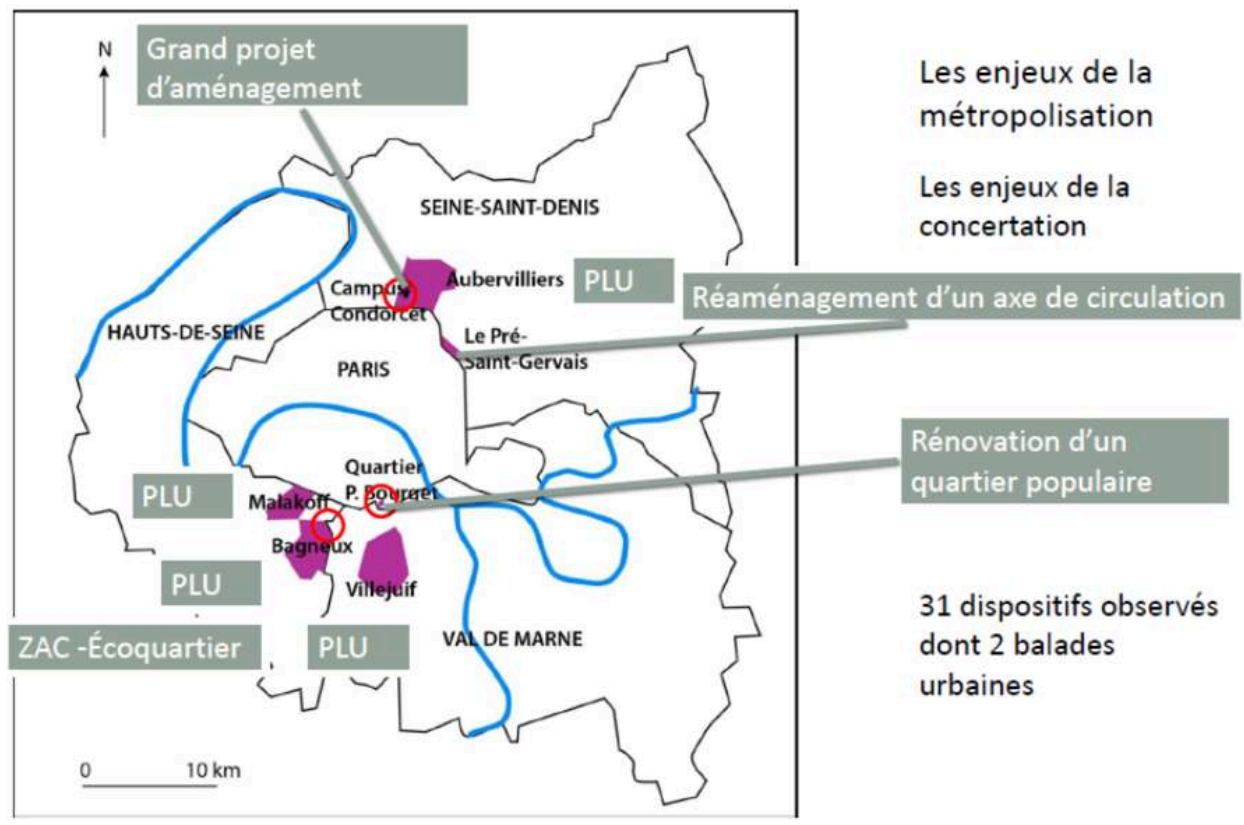

Source : H. Chelzen

3 La figure 2 témoigne de la méthodologie innovante déployée pour analyser les échanges : les couleurs représentent les différentes catégories d'acteurs. L'alternance des couleurs correspond à l'alternance des prises de parole par les catégories d'acteurs. Le déroulement du temps et le temps de parole de chaque catégorie d'acteurs sont à lire horizontalement. Les lignes correspondent aux thématiques abordées durant la réunion. Les propos des participants ont été retranscrits et classés selon ces thématiques. L'analyse des propos des participants en fonction de ces thématiques permet de procéder à un traitement qualitatif et quantitatif. Ainsi, au-delà des projets soumis au débat, c'est le territoire qui se révèle et s'affirme comme un espace approprié 
de manière différente selon la diversité les catégories d'acteurs. En effet, le territoire est vecteur d'une pluralité de représentations: images idéelles, images graphiques comme les cartes, les photographies, les images du projet.

Figure 2. Le déroulement d'une réunion publique, Bagneux, 11.03.2011, $116 \mathrm{mn}$

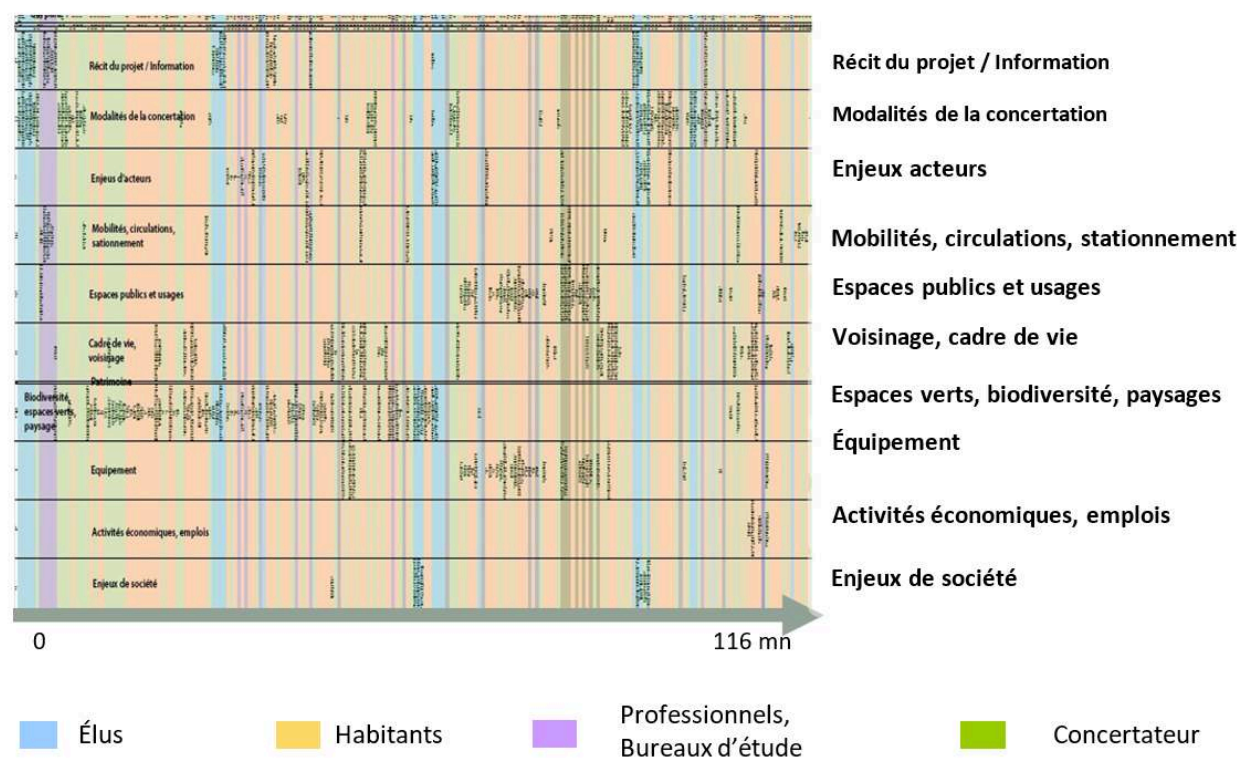

Source : H. Chelzen

Sept réunions ont été retranscrites dans des tableaux Excel. Pour analyser ces tableaux, le concept de dissonance est mobilisé. Ce terme est utilisé par opposition au sens commun de la concertation et désigne une rupture l'harmonie de l'accord. Trois types de dissonances se dessinent : la dissonance participative, la dissonance discursive et la dissonance territoriale. La dissonance participative rend compte de l'évaluation différenciée du dispositif participatif par les catégories d'acteurs. Cette évaluation de la participation s'appuie sur les paliers de la participation (figure 3) et se fonde sur des données objectives, le calcul des temps de parole. 
Figure 3. L'évaluation de la participation

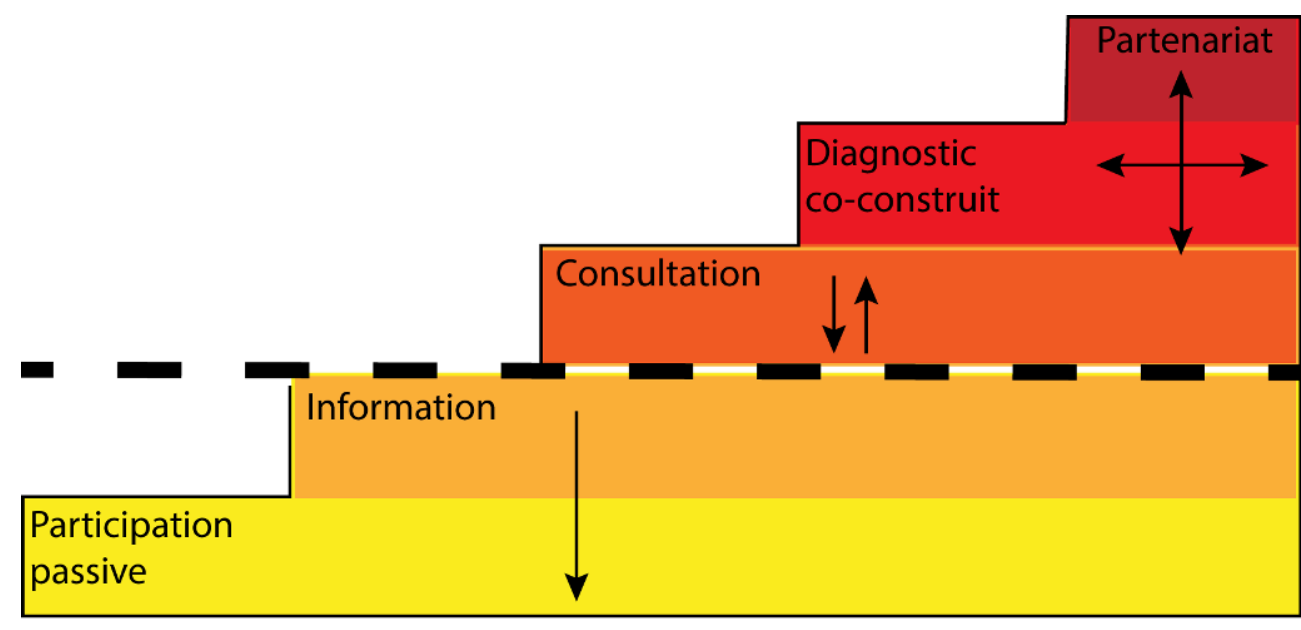

Tensions, controverses, conflits

Source : H. Chelzen

La dissonance discursive s'appuie sur l'analyse des préoccupations des habitants et des porteurs de projet, à partir du calcul du temps de parole des catégories d'acteurs par thématiques abordées. L'information du projet est davantage abordée par les porteurs de projet, tandis que les modalités de la concertation ou la question des mobilités et des circulations sont davantage nourries par les habitants. Les préoccupations des uns et des autres se correspondent rarement. Enfin, la dissonance territoriale rend compte du décalage des acteurs en présence concernant les pratiques, les perceptions et les représentations du territoire. Par exemple, lorsque l'environnement et la biodiversité sont en jeu ou, lorsque la carte du projet agit comme un argument performatif, la dissonance territoriale est forte.

6 À l'issue de ces enquêtes, il apparaît que les porteurs de projets ne peuvent faire la ville sans les habitants ni pour eux : désormais, ils doivent faire la ville avec les habitants.

\author{
Discipline \\ Géographie \\ Directeur \\ Pierre Pech \\ Université \\ Université Paris 1 Panthéon-Sorbonne ; Ladyss UMR 7533 \\ Membres du jury de thèse, soutenue le 25/04/2018 \\ Cécile BLATRIX, Professeure, AgroParis Tech, rapportrice \\ Catherine CARRÉ, Professeure, Université Paris I Panthéon-Sorbonne, \\ examinatrice \\ Guillaume FABUREL, Professeur, Université Lyon II, examinateur \\ Anne JÉGOU, Maîtresse de Conférences, Université de Franche-Comté, \\ examinatrice
}


Pierre PECH, Professeur, Université Paris I Panthéon-Sorbonne, directeur de thèse Martin VANIER, Professeur, École d'Urbanisme de Paris, rapporteur

Situation professionnelle actuelle

Enseignante dans le secondaire. Chercheure associée au Ladyss - UMR 7533.

Courriel de l'auteur

mailto: lnchelzen[at]gmail.com

INDEX

Thèmes : Carnets de soutenances 